$01.1 ; 13.1 ; 13.3$

\title{
Функционализация поверхности low-k диэлектриков ионами Ar низкой энергии
}

\author{
(C) А.А. СоловыХ ${ }^{1}$, А.А. Сычева ${ }^{2}$, Е.Н. Воронина ${ }^{1,2}$ \\ ${ }^{1}$ Московский государственный университет им. М.В. Ломоносова, Москва, Россия \\ ${ }^{2}$ Научно-исследовательский институт ядерной фризики им. Д.В. Скобельцына Московского государственного \\ университета им. М.В. Ломоносова, \\ Москва, Россия \\ E-mail: sycheva.phys@gmail.com
}

Поступило в Редакцию 19 ноября 2021г.

В окончательной редакции 13 января 2022 г.

Принято к публикации 16 января 2022г.

Представлены результаты моделирования воздействия атомов и ионов Ar низкой энергии на поверхность $l o w-k$ диэлектрика с использованием метода теории функционала плотности. Исследован механизм удаления $\mathrm{CH}_{3}$-групп с поверхности материала под воздействием атомов и ионов Ar. Получены оценки пороговой энергии данного процесса.

Ключевые слова: low- $k$ диэлектрики, функционализация, компьютерное моделирование, диффузионные барьеры, метильные группы.

DOI: 10.21883/PJTF.2022.07.52286.19085

В настоящее время нанопористые органосиликатные пленки с ультранизкой диэлектрической проницаемостью $k$ (low- $k$ диэлектрики) на основе аморфных матриц $\mathrm{SiO}_{x}$ активно применяются для изоляции медных проводников в межслойных соединениях современных сверхбольших интегральных схем [1]. Однако подобная технология приводит к возникновению ряда проблем, одна из которых связана с диффузией атомов $\mathrm{Cu}$ в глубь $l o w-k$ пленки, что приводит к ухудшению диэлектрических свойств материала. Для предотвращения возникновения данного эффекта на поверхность создаваемых в low- $k$ пленках узких тренчей наносят ультратонкие (до $5 \mathrm{~nm}$ ) барьеры из тугоплавких металлов [1,2], но этому препятствует сложная морфология low- $k$ диэлектриков, низкие значения $k$ которых достигаются за счет наличия в их структуре пор, покрытых гидрофобными $\mathrm{CH}_{3}$-группами. Для усиления адгезии атомов металла с диэлектриком необходимо провести предварительную обработку тонкого приповерхностного слоя $l o w-k$ материалов путем удаления $\mathrm{CH}_{3}$-групп, т.е. функционализацию. Подобная обработка, проводимая в плазме, может приводить к деградации свойств $l o w-k$ пленок в результате воздействия химически активных радикалов и вакуумного ультрафиолетового излучения. Поэтому для функционализации поверхности этих материалов планируется использовать ионы низкой (до $20 \mathrm{eV}$ ) энергии, которые способны удалять метильные группы не только со дна, но и с боковых стенок тренчей [3], не вызывая заметных существенных повреждений в нижележащих слоях диэлектрика.

Цель настоящей работы состоит в изучении воздействия атомов и ионов $\mathrm{Ar}$ низкой энергии (менее $20 \mathrm{eV}$ ) на $l o w-k$ диэлектрик и выявлении условий, при которых возможно удаление метильных групп с его поверхности, с использованием квантово-механического метода теории функционала плотности (DFT). В отличие от метода Хартри-Фока в стационарном методе DFT полная энергия основного состояния системы представляется в виде функционала не от многоэлектронной волновой функции, а от электронной плотности системы [4]. Метод DFT не только является более эффективным с точки зрения вычислительных затрат, но и позволяет во многих случаях получать более точные результаты за счет введения приближенного функционала, описывающего обменные и корреляционные эффекты. В настоящей работе применялся обменно-корреляционный функционал PBE в рамках обобщенного градиентного приближения GGA, который обеспечивает корректное описание взаимодействия атомов и ионов инертных газов с различными химическими элементами в широком диапазоне энергий [5]. Динамические DFT-расчеты с использованием алгоритмов молекулярной динамики выполнялись в базисе плоских волн с псевдопотенциалами PAW [6] в программном пакете Vienna Ab Initio Simulation Package (VASP) [7] на суперкомпьютерном комплексе МГУ им. М.В. Ломоносова [8]. С целью снижения вычислительных затрат в качестве модели low-k диэлектрика была выбрана молекула ПОСС (полиэдральные олигомерные силсесквиоксаны) с присоединенной к ней метильной группой, описываемая химической формулой $\mathrm{Si}_{8} \mathrm{O}_{12} \mathrm{H}_{7}-\mathrm{CH}_{3}$ (рис. 1). Подобные упрощенные молекулярные модели, содержащие характерные для low-k диэлектриков связи $\mathrm{Si}-\mathrm{O}$ и $\mathrm{Si}-\mathrm{C}$, активно применяются для изучения реакций на поверхности этих материалов [9]. В динамических расчетах варьировались начальная энергия налетающих частиц $E_{0}=5-20 \mathrm{eV}$ и 


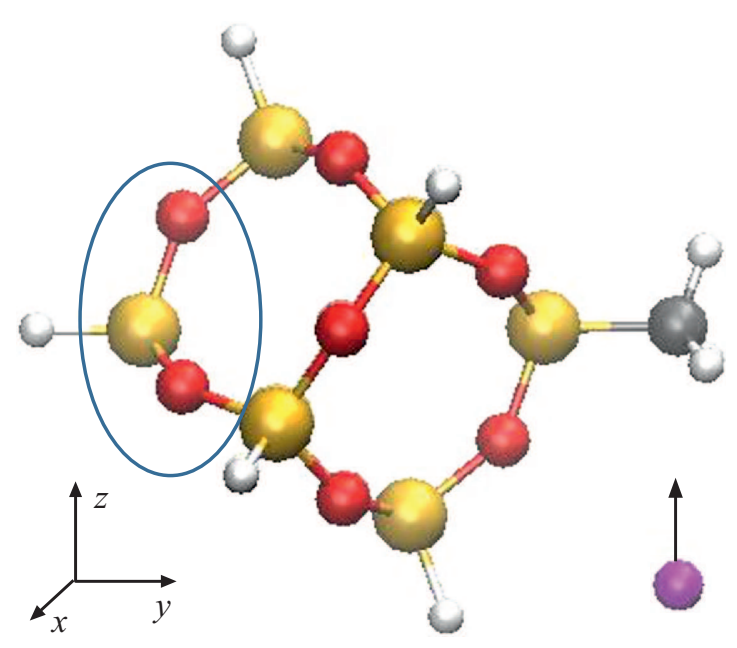

Рис. 1. Взаимное расположение модели молекулы ПОСС и налетающего атома/иона $\mathrm{Ar}$, при котором было получено минимальное значение пороговой энергии. Направление движения налетающей частицы показано стрелкой. Линией обведены атомы, которые были зафиксированы для предотвращения поступательного и вращательного движения молекулы.

углы между направлением начальной скорости $\mathrm{Ar}$ и линией связи $\mathrm{Si}-\mathrm{C}$, а также рассматривались различные прицельные расстояния до атома С. Нижняя граница диапазона энергий $E_{0}$ была определена на основании предварительного статического расчета энергии связи $\mathrm{Si}-\mathrm{C}$ в молекуле ПОСС, полученное значение $4.4 \mathrm{eV}$ - хорошо согласуется с имеющимися экспериментальными данными (см., например, [10]). Временной шаг был выбран равным $0.1 \mathrm{fs}$ при длительности одной траектории 0.5-1.0 ps. Для визуализации и анализа расчетных данных использовался пакет VMD [11].

Результаты динамического моделирования показали, что пороговая энергия иона $E_{t h}$, при которой наблюдается удаление $\mathrm{CH}_{3}$-группы, существенно зависит от геометрии взаимодействия. Минимальное значение $E_{t h}=11 \mathrm{eV}$ было получено при подлете $\mathrm{Ar}$ непосредственно к атому С под углом 90 (рис. 1). Выполненный анализ расчетных данных позволил выявить основные особенности механизма процесса удаления метильной группы. На рис. 2, $a$ показаны последовательные изменения положений атомов в молекуле ПОСС и налетающего атома $\mathrm{Ar}$ для одной из рассчитанных траекторий в случае, когда происходил вылет радикала $\mathrm{CH}_{3}$, а на рис. 2,b - временны́е зависимости кинетической энергии атома $\mathrm{Ar}$ и радикала при различных значениях начальной энергии $E_{0}$. Как видно из представленных данных, атомы Ar теряют значительную долю $(\sim 80 \%)$ своей исходной энергии $E_{0}$ в результате взаимодействия с молекулой ПОСС (кривые $1-3$ на рис. $2, b)$ и отклоняются от первоначального направления на угол $\sim 35-40^{\circ}$, который уменьшается с ростом $E$. Значительная часть $(\sim 50-60 \%)$ энергии, передаваемой молекуле, переходит в кинетическую энер- гию метильной группы $E_{\mathrm{CH}_{3}}$, которая быстро возрастает после соударения, а затем снижается и приближается к некоторому постоянному значению на асимптотике (кривые 4-6 на рис. 2,b). Если переданной энергии недостаточно для разрыва связи $\mathrm{Si}-\mathrm{C}$, наблюдаются колебания метильной группы, медленно затухающие в процессе релаксации молекулы (кривая 6 на рис. 2,b). В противном случае происходит вылет радикала $\mathrm{CH}_{3}$, который испытывает притяжение со стороны молекулы ПОСС на относительно малых расстояниях от нее, что вызывает уменьшение его кинетической энергии. Итоговое значение $E_{\mathrm{CH}_{3}}$ на асимптотике примерно соответствует разности энергии, переданной метильной группе, и энергии связи $\mathrm{Si}-\mathrm{C}$ (кривые 4 и 5 на рис. $2, b)$.

Изменение угла подлета налетающей частицы или области удара приводит к возрастанию пороговой энергии (например, при воздействии под углом $60^{\circ}$ к связи $\mathrm{Si}-\mathrm{C}$ величина $E_{t h}$ увеличивается до $\left.\sim 17.5 \mathrm{eV}\right)$. При этом доля энергии, переданной $\mathrm{CH}_{3}$-группе, снижается до $\sim 40 \%$, а в молекуле ПОСС наблюдаются заметные колебания атомов вблизи траектории движения Ar, приводящие к более существенной деформации ее структуры, чем в случае удара перпендикулярно связи $\mathrm{Si}-\mathrm{C}$. Для выявления изменений в электронном строении молекулы ПОСС до и после взаимодействия с нейтральным атомом Ar был выполнен анализ заселенностей, результаты которого приведены в таблице, и рассчитано пространственное распределение спиновой электронной плотности (вставки на рис. 2,a). Хорошо видно, что в результате отрыва метильной группы положительный заряд на атоме $\mathrm{Si}$ в молекуле ПОСС уменьшается, а спиновая плотность оказывается локализована на этом атоме $\mathrm{Si}$ и на радикале $\mathrm{CH}_{3}$. Таким образом, полученные результаты показывают, что при воздействии нейтрального атома $\mathrm{Ar}$ на метильную группу столкновительный механизм взаимодействия играет основную роль, а структурные изменения в молекуле ПОСС оказываются незначительными.

Представленные выше данные были получены в результате моделирования взаимодействия нейтрального атома $\mathrm{Ar}$ с молекулой ПОСС. Для исследования воздействия положительно заряженного иона $\mathrm{Ar}$ в дальнейших расчетах заряд задавался для всей системы в связи с особенностями реализации метода DFT в VASP. Результаты динамического моделирования, представленные на рис. 2, $c, d$ и в таблице, наглядно демонстрируют различия в механизме воздействия иона по сравнению с нейтральным атомом. В отличие от предыдущего случая в результате удара происходит заметное изменение геометрии молекулы ПОСС: атом $\mathrm{Si}$ смещается по направлению к центру молекулы, что приводит к изменению длин связей $\mathrm{Si}-\mathrm{O}$ и валентных углов $\mathrm{O}-\mathrm{Si}-\mathrm{O}$ (см. таблицу). Анализ временны́х зависимостей кинетической энергии в случае воздействия иона $\operatorname{Ar}$ (рис. 2,d) показывает, что вся энергия, переданная ионом $\mathrm{CH}_{3}$-группе, не уменьшается после 

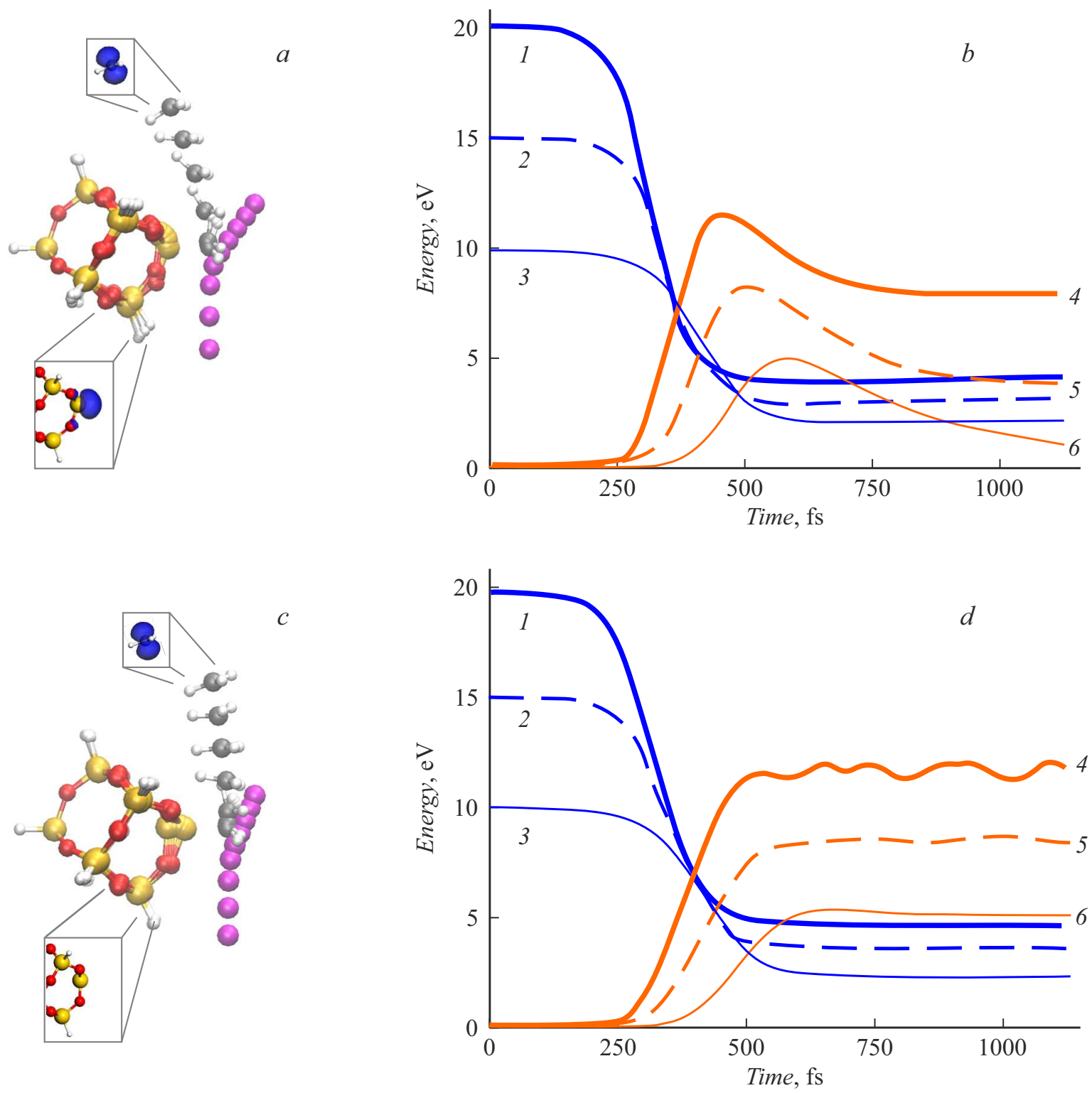

Рис. 2. $a, c$ - последовательные изменения положений атомов в молекуле ПОСС и налетающего $\operatorname{Ar}\left(E_{0}=12.5 \mathrm{eV}\right)$ без учета заряда $(a)$ и с учетом заряда $(c)$, отмеченные каждые $10 \mathrm{fs}$ в течение первых $140 \mathrm{fs}$ моделирования. На вставках синим цветом показано пространственное распределение спиновой электронной плотности (для значения $0.05 \AA^{-3}$ ) в молекуле ПОСС и $\mathrm{CH}_{3}$ радикале (цветной вариант рисунка представлен в электронной версии статьи). $b, d-$ временны́е зависимости кинетической энергии $\operatorname{Ar}(1-3)$ и вылетающего $\mathrm{CH}_{3}$-радикала $(4-6)$ для $E_{0}=10(3,6), 15(2,5), 20 \mathrm{eV}(1,4)$ без учета заряда $(b)$ и с учетом заряда $(d)$.

Параметры молекулы ПОСС до и после воздействия $\operatorname{Ar}(R-$ длина связи, $\alpha-$ валентный угол, $Q-$ частичный заряд атома, $B O$ - величина интеграла перекрытия, характеризующего прочность связи)

\begin{tabular}{|c|c|c|c|c|c|c|c|c|c|c|}
\hline \multirow[b]{3}{*}{$\begin{array}{c}\text { До } \\
\text { взаимодействия }\end{array}$} & \multirow[b]{3}{*}{-} & \multicolumn{2}{|c|}{$R, \AA$} & \multicolumn{2}{|c|}{$\alpha$, deg } & \multicolumn{2}{|c|}{$Q, e$} & \multicolumn{3}{|c|}{$B O$} \\
\hline & & $\mathrm{Si}-\mathrm{O}$ & $\mathrm{C}-\mathrm{H}$ & $\mathrm{O}-\mathrm{Si}-\mathrm{O}$ & $\mathrm{H}-\mathrm{C}-\mathrm{H}$ & $\mathrm{Si}$ & $\mathrm{O}$ & $\mathrm{Si}-\mathrm{O}$ & $\mathrm{Si}-\mathrm{C}$ & $\mathrm{C}-\mathrm{H}$ \\
\hline & & 1.65 & 1.10 & 109.35 & 108.3 & +1.77 & -0.93 & 0.58 & 0.81 & 0.73 \\
\hline $\begin{array}{c}\text { После вылета } \\
\mathrm{CH}_{3} \text {-группы }\end{array}$ & Без заряда & 1.65 & 1.10 & 109.67 & 120.0 & +1.35 & -0.91 & 0.81 & - & 0.73 \\
\hline & С зарядом & 1.58 & 1.10 & 119.92 & 120.0 & +1.75 & -0.87 & 0.76 & - & 0.73 \\
\hline
\end{tabular}


вылета, т.е. она не испытывает притяжения со стороны молекулы. Это обусловливает существенное снижение величины $E_{t h}$ : удаление метильной группы наблюдается при воздействии иона $\mathrm{Ar}$ с начальной энергией $\sim 5 \mathrm{eV}$. На основании анализа расчетных данных можно сделать вывод, что воздействие иона на метильную группу приводит к его нейтрализации и возникновению в молекуле ПОСС дефекта с локализованным на нем избыточным положительным зарядом, что подтверждается рассчитанными частичными зарядами на атомах (см. таблицу). Обнаруженный зарядовый дефект может быстро нейтрализоваться налетающим потоком электронов на дне тренчей, однако на боковых стенках он способен сохраняться значительно дольше.

Важно, что приведенные выше значения пороговой энергии $E_{t h}$ были получены для оптимальных параметров воздействия аргона. Однако поверхность $l o w-k$ диэлектриков не является идеально ровной, поэтому при облучении материала взаимодействие налетающих частиц с $\mathrm{CH}_{3}$-группами на боковых стенках тренчей может происходить под различными углами к связи $\mathrm{Si}-\mathrm{C}$, что приводит к повышению $E_{t h}$. Также следует учитывать, что при нейтрализации иона вблизи поверхности может выделяться дополнительная энергия, поэтому для определения точного значения $E_{t h}$ иона для отрыва метильной группы требуются расчеты с помощью более точных $a b$ initio методов.

Таким образом, выполненное моделирование показало, что воздействие атомов и ионов $\mathrm{Ar}$ на поверхность low- $k$ диэлектрика может приводить к разрыву связи $\mathrm{Si}-\mathrm{C}$ и последующему удалению $\mathrm{CH}_{3}$-группы. Пороговая энергия атома Ar, при которой происходит вылет $\mathrm{CH}_{3}$-группы, принимает минимальное значение $\sim 11 \mathrm{eV}$ в случае воздействия на атом С под углом 90 связи $\mathrm{Si}-\mathrm{C}$. Наличие положительного заряда у налетающей частицы приводит к отрыву метильной группы даже при энергии $5 \mathrm{eV}$ при оптимальной траектории воздействия. Таким образом, ионы $\mathrm{Ar}$ с ультранизкой энергией 5-20 eV способны удалять гидрофобные $\mathrm{CH}_{3}$ группы в поверхностном слое $l o w-k$ пленок. Полученные результаты могут быть использованы для разработки методики предварительной функционализации поверхности $l o w-k$ диэлектриков потоками ионов низкой энергии с целью последующего создания диффузионных барьеров.

\section{Финансирование работы}

Работа выполнена при поддержке Российского фонда фундаментальных исследований (грант № 18-29-27001).

\section{Список литературы}

[1] M.R. Baklanov, P.S. Ho, E. Zschech, Advanced interconnects for ULSI technology (Wiley \& Sons, 2012).

[2] H. Xu, Zh.-J. Hu, X.-P. Qu, H. Wan, Sh.-S. Yan, M. Li, Sh.-M. Chen, Yu-H. Zhao, J. Zhang, M.R. Baklanov, Appl. Surf. Sci., 498, 143887 (2019).

DOI: $10.1016 / j$.apsusc.2019.143887

[3] A.P. Palov, O.V. Proshina, T.V. Rakhimova, A.T. Rakhimov, E.N. Voronina, Plasma Process. Polym., 18 (7), 2100007 (2021). DOI: $10.1002 /$ ppap.202100007

[4] В. Кон, УФН, 172 (3), 336 (2002). DOI: $10.3367 /$ UFNr.0172.200203e.0336

[5] A.N. Zinoviev, K. Nordlund, Nucl. Instrum. Meth. Phys. Res. B, 406, 511 (2017). DOI: 10.1016/j.nimb.2017.03.047

[6] P.E. Blöchl, Phys. Rev. B, 50 (24), 17953 (1994). DOI: 10.1103/PhysRevB.50.17953

[7] G. Kresse, D. Joubert, Phys. Rev. B, 59 (3), 1758 (1999). DOI: 10.1103/PhysRevB.59.1758

[8] V.V. Voevodin, A.S. Antonov, D.A. Nikitenko, P.A. Shvets, S.I. Sobolev, I.Yu. Sidorov, K.S. Stefanov, V.V. Voevodin, S.A. Zhumatiy, Supercomput. Front. Innov., 6 (2), 4 (2019). DOI: $10.14529 /$ jsfi190201

[9] E.N. Voronina, Yu.A. Mankelevich, T.V. Rakhimova, D.V. Lopaev, J. Vac. Sci. Technol. A, 37 (6), 061304 (2019). DOI: $10.1116 / 1.5122655$

[10] B. deB. Darwent, Bond dissociation energies in simple molecules, Nat. Stand. Ref. Data Ser. (Nat. Bur. Stand., Washington, 1970).

[11] W. Humphrey, A. Dalke, K. Schulten, J. Mol. Graphics, 14, 33 (1996). DOI: 10.1016/0263-7855(96)00018-5

\section{Конфликт интересов}

Авторы заявляют, что у них нет конфликта интересов. 